\title{
Initiality for Typed Syntax and Semantics
}

\author{
Benedikt Ahrens \\ Université Nice Sophia Antipolis, France
}

\begin{abstract}
We give an algebraic characterization of the syntax and semantics of a class of simply-typed languages, such as the language PCF: we characterize simply-typed binding syntax equipped with reduction rules via a universal property, namely as the initial object of some category. For this purpose, we employ techniques developed in two previous works: in [2], we model syntactic translations between languages over different sets of types as initial morphisms in a category of models. In [1], we characterize untyped syntax with reduction rules as initial object in a category of models. In the present work, we show that those techniques are modular enough to be combined: we thus characterize simply-typed syntax with reduction rules as initial object in a category. The universal property yields an operator which allows to specify translations - that are semantically faithful by construction — between languages over possibly different sets of types.

We specify a language by a 2-signature, that is, a signature on two levels: the syntactic level specifies the types and terms of the language, and associates a type to each term. The semantic level specifies, through inequations, reduction rules on the terms of the language. To any given 2 -signature we associate a category of models. We prove that this category has an initial object, which integrates the types and terms freely generated by the 2-signature, and the reduction relation on those terms generated by the given inequations. We call this object the (programming) language generated by the 2-signature.
\end{abstract}

\section{Introduction}

We give a characterization, via a universal property, of the syntax and semantics of simply-typed languages with variable binding. More precisely, we characterize the terms and sorts associated to a signature equipped with reduction rules as the initial object in a category of models. Initiality in this category gives rise to an iteration principle (cf. Rem. 45) which allows to specify translations between languages in a convenient way as initial morphisms. The category of models is sufficiently large - and thus the iteration principle stemming from initiality is sufficiently general - to account for translations between languages over different sets of sorts. Furthermore, translations specified via this principle are ensured to be faithful with respect to reduction in the source and target languages, as well as compatible in a suitable sense with substitution on either side.

To illustrate the iteration operator stemming from initiality, we use it to specify a translation from PCF to the untyped lambda calculus ULC. We do so in 
the proof assistant Coq [5]; for this purpose, we prove formally, in Coq, an instance of our main theorem for the 2-signature of PCF: the types and terms of PCF, equipped with their usual reductions, form an initial object in the category of models of PCF. We then use the iteration principle to obtain an initial morphism - a translation, faithful with respect to reductions - to ULC, as an executable Coq function. The Coq theory files as well as documentation are available online at http://math.unice.fr/laboratoire/logiciels.

Summary We define a notion of 2-signature which allows the specification of the types and terms of a language - via an underlying 1-signature - as well as its semantics in form of reduction rules. A 1 -signature $(S, \Sigma)$ is given by a pair of a signature $S$ for types and a binding signature $\Sigma$ for terms typed over the set of types associated to $S$. Reduction rules for terms generated by $\Sigma$ are specified via a set $A$ of inequations over $(S, \Sigma)$. A 2 -signature $((S, \Sigma), A)$ is a pair of a 1-signature $(S, \Sigma)$ and a set $A$ of inequations over $(S, \Sigma)$. To such a 2 -signature we associate a category of representations, for which the types and terms generated by $(S, \Sigma)$, equipped with reductions according to $A$, forms an initial object.

1-signatures are defined in [2]. There, we associate a category $\operatorname{Rep}(S, \Sigma)$ of representations to any 1 -signature $(S, \Sigma)$, and show that the types and terms freely generated by $(S, \Sigma)$ form an initial object in this category. Representations there are built from monads on families of sets. In the present work, we build a different category $\operatorname{Rep}^{\Delta}(S, \Sigma)$ of representations using relative monads from sets to preordered sets, which allows - in a second step, cf. below - the integration of reduction rules to account for semantic aspects. The two categories of representations, $\operatorname{Rep}(S, \Sigma)$ and $\operatorname{Rep}^{\Delta}(S, \Sigma)$, are connected through an adjunction which transports the initial object of the former to the latter category (cf. Lem. 34).

Inequations over untyped 1-signatures are considered in [1]. There, we define a notion of 2-signature for untyped syntax with semantics in form of reduction rules and show that its associated category of representations has an initial object. In the present work, we define inequations over typed 1-signatures as defined in [2]. Given a set $A$ of inequations over a 1 -signature $(S, \Sigma)$, the representations of $(S, \Sigma)$ that satisfy each inequation of $A$, form a full subcategory of $\operatorname{Rep}^{\Delta}(S, \Sigma)$, which we call the category of representations of $(S, \Sigma, A)$. Our main theorem (cf. Thm. 44) states that this category has an initial object, which integrates the types and terms freely generated by $(S, \Sigma)$, equipped with reduction rules generated by the inequations of $A$.

Related Work Related work is reviewed extensively in [1,2], as well as in the author's $\mathrm{PhD}$ thesis [3]. We give a brief overview: rewriting in nominal settings is examined by Férnandez and Gabbay [6]. Ghani and Lüth [8] present rewriting for algebraic theories without variable binding; they characterize equational theories resp. rewrite systems as coequalizers resp. coinserters in a category of monads on the categories Set resp. Pre. Fiore and Hur [7] have extended Fiore's work to integrate semantic aspects into initiality results. In particular, Hur's thesis 
[12] is dedicated to equational systems for syntax with variable binding. In a "Further research" section [12, Chap. 9.3], Hur suggests the use of preorders, or more generally, arbitrary relations to model inequational systems. Hirschowitz and Maggesi [9] prove initiality of the set of lambda terms modulo beta and eta conversion in a category of exponential monads. In an unpublished paper [10], they define a notion of half-equation and equation to express congruence between terms. We adopt their definition in this paper, but interpret a pair of half-equations as inequation rather than equation.

\section{Relative Monads and Modules}

Relative monads were defined by Altenkirch et al. [4] to overcome the restriction of (regular) monads to endofunctors. In an earlier work [1], we define morphisms of relative monads and modules over relative monads. In the following section we define a more general notion of colax morphism of relative monads - which we use in Sect. 3 to model translations between languages over different sets of types - and generalize constructions of [1] to such colax morphisms. Some definitions from [1,2] which we use in the present work, are recalled at the beginning.

We denote by Set the category of sets and total maps of sets. We call Pre the category of preordered sets and monotone maps between them.

Definition 1. We call $\Delta$ : Set $\rightarrow$ Pre the left adjoint of the forgetful functor $U:$ Pre $\rightarrow$ Set. The functor $\Delta$ associates to each set $X$ the set itself together with the smallest preorder, i.e. the diagonal of $X, \Delta(X):=\left(X, \delta_{X}\right)$.

Definition 2 (Category of Families). Let $\mathcal{C}$ be a category and $T$ be a set, i.e. a discrete category. We denote by $\mathcal{C}^{T}$ the functor category, an object of which is a $T$-indexed family of objects of $\mathcal{C}$. We write $V_{t}:=V(t)$ for objects and morphisms. Given a functor $F: \mathcal{C} \rightarrow \mathcal{D}$, we denote by $F^{T}: \mathcal{C}^{T} \rightarrow \mathcal{D}^{T}$ the induced functor.

Definition 3 (Relative Monad on $\Delta^{T}$, enriched). We strengthen the definition of a relative monad $P$ on $\Delta^{T}$ by requiring the substitution map $\sigma_{X, Y}$ to be monotone with respect to the preorders induced by the preorders on PY,

$$
\sigma_{X, Y}: \operatorname{Pre}^{T}(\Delta X, P Y) \rightarrow \operatorname{Pre}^{T}(P X, P Y) .
$$

From now on, a relative monad on $\Delta^{T}$ is meant to be enriched in the sense of Def. 3, i.e. monotone in both the first- and the higher-order argument.

Example 4 (Lambda Calculus as Relative Monad on $\Delta^{T}$ ). Let $T:=T_{\mathrm{TLC}}$ be the set of types of the simply-typed lambda calculus, built from a base type and a binary arrow constructor. Given a set family $V \in \mathrm{Set}^{T_{\mathrm{TLC}}}$, we denote by $\operatorname{TLC}(V) \in$ Set $^{T_{\mathrm{TLC}}}$ the set family of simply-typed lambda terms over $T_{\mathrm{TLC}}$ in context $V$, which might be implemented in the proof assistant Coq as follows:

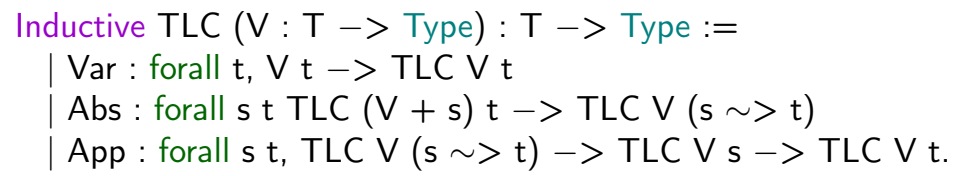


Here $\mathrm{V}+\mathrm{s}$ is a notation denoting the context $\mathrm{V}$ extended by a fresh variable of type $s$ - the variable that is bound by the constructor Abs $s$ t. We occasionally leave the object type arguments of the constructors implicit and write $\lambda M$ and $M(N)$ for Abs s t $\mathrm{M}$ and App s t $\mathrm{M} \mathrm{N}$, respectively. The set family of lambda terms is equipped with a structure of a monad TLC on the category Set ${ }^{T_{T L C}}$ as follows [11]: the family $\eta^{\mathrm{TLC}}$ is given by the family of constructors Var, and the substitution map is given by capture-avoiding simultaneous substitution:

$$
\sigma_{X, Y}: \operatorname{Set}^{T}(X, \operatorname{TLC}(Y)) \rightarrow \operatorname{Set}^{T}(\operatorname{TLC}(X), \operatorname{TLC}(Y)) .
$$

Similarly, with the same operations $\eta$ and $\sigma$, we can consider it as a relative monad on the functor $\Delta^{T \text { TLC }}$,

$$
\mathrm{TLC} \Delta: \mathrm{Set}^{T_{\mathrm{TLC}}} \rightarrow \mathrm{Pre}^{T_{\mathrm{TLC}}} .
$$

The underlying object map TLC $\Delta$ associates, to each set family $V$, the family of lambda terms in context $V$, equipped with the diagonal preorder, corresponding to syntactic equality:

$$
\mathrm{TLC} \Delta: V \mapsto\left(\operatorname{TLC}(V), \delta_{\mathrm{TLC}(V)}\right) .
$$

We equip each set $\operatorname{TLC}(V)(t)$ of lambda terms over context $V$ of object type $t$ with a preorder taken as the reflexive-transitive closure of the relation generated by the beta rule

$$
\lambda M(N) \leq M[*:=N]
$$

and its propagation into subterms:

$$
\operatorname{TLC} \beta: V \mapsto\left(\operatorname{TLC}(V), \beta_{\operatorname{TLC}(V)}^{*}\right) .
$$

The beta rule in Disp. $(\beta)$ states that the application of a lambda abstraction with body $M$ to an argument $N$ reduces to the term $M$ in which the term $N$ is substituted for the fresh variable of $M$ - recall from above that $M$ lives in an extended context - in a capture-avoiding manner. This assignment defines a relative monad TLC $\beta$ on the functor $\Delta^{T}: \mathrm{Set}^{T} \rightarrow \operatorname{Pre}^{T}$.

Modules over relative monads and their morphisms are defined in [1], together with several constructions of modules. Recall that modules over $P$ with codomain $\mathcal{E}$ and morphisms between them form a category called $\operatorname{RMod}(P, \mathcal{E})$. We give some examples of modules and module morphisms over the monad TLC $\beta$, which hold analogously for the monad TLC $\Delta$ :

Example 5 (Ex. 4 cont.). The map TLC $\beta: V \mapsto \operatorname{TLC} \beta(V)$ yields a module over the relative monad $\operatorname{TLC} \beta$, the tautological TLC $\beta$-module $\operatorname{TLC} \beta$. Given $V \in \operatorname{Set}^{T}$ and $s \in T$, we denote by $V^{s}$ the context $V$ enriched by an additional variable of type $s$. The map TLC $\beta^{s}: V \mapsto \operatorname{TLC} \beta\left(V^{s}\right)$ inherits the structure of a TLC $\beta$-module from the tautological module $\operatorname{TLC} \beta$. We call $\mathrm{TLC}^{s}$ the derived module with respect to $s \in T$ of the module TLC $\beta$. Given $t \in T$, the map $V \mapsto \operatorname{TLC} \beta(V)(t): \mathrm{Set}^{T} \rightarrow$ 
Pre inherits a structure of a $\operatorname{TLC} \beta$-module, the fibre module $[\mathrm{TLC} \beta]_{t}$ with respect to $t \in T$. Given $s, t \in T$, the map $V \mapsto \operatorname{TLC} \beta(V)(s \rightsquigarrow t) \times \operatorname{TLC} \beta(V)(s)$ inherits a structure of a TLC $\beta$-module. Finally, the constructors of abstraction Abs s t and application App s $\mathrm{t}$ are carriers of morphisms of TLC $\beta$-modules:

$$
\mathrm{Abs}_{s, t}:\left[\mathrm{TLC} \beta^{s}\right]_{t} \rightarrow[\mathrm{TLC} \beta]_{s \rightsquigarrow t}, \quad \mathrm{App}_{s, t}:[\mathrm{TLC} \beta]_{s \rightsquigarrow t} \times[\mathrm{TLC} \beta]_{s} \rightarrow[\mathrm{TLC} \beta]_{t} .
$$

Analogous remarks hold for the monad TLC $\Delta$ and modules over this monad.

As in Ex. 4, we consider a language over a set $T$ of types as a (relative) monad on $\Delta^{T}$. Translations between languages are given by colax morphisms of monads:

Definition 6. Suppose given two relative monads $P: \mathcal{C} \stackrel{F}{\rightarrow} \mathcal{D}$ and $Q: \mathcal{C}^{\prime} \stackrel{F^{\prime}}{\rightarrow} \mathcal{D}^{\prime} . A$ colax morphism of relative monads from $P$ to $Q$ is a quadruple $h=\left(G, G^{\prime}, N, \tau\right)$ of a functor $G: \mathcal{C} \rightarrow \mathcal{C}^{\prime}$, a functor $G^{\prime}: \mathcal{D} \rightarrow \mathcal{D}^{\prime}$ as well as a natural transformation $N: F^{\prime} G \rightarrow G^{\prime} F$ and a natural transformation $\tau: P G^{\prime} \rightarrow G Q$ such that the following diagrams commute for any objects $c, d$ and any suitable morphism $f$ :
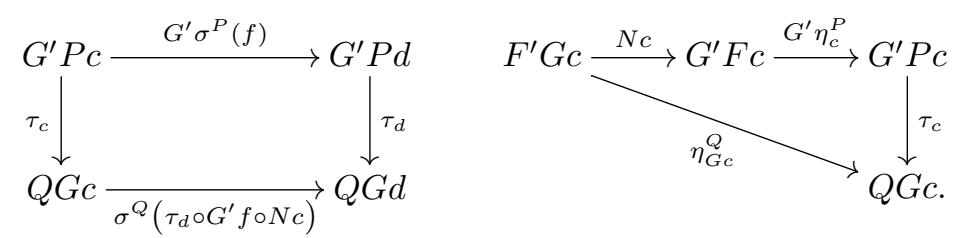

Given a morphism of relative monads $h: P \rightarrow Q$ and a $Q-$ module $N$ with codomain $\mathcal{E}$, we define the pullback $P$-module $h^{*} N$, also with codomain $\mathcal{E}$ :

Definition 7. We define the pullback of $N$ along $h$ with object map $c \mapsto M(G c)$ and with substitution map, for $f: F c \rightarrow P d$, as $\varsigma^{h^{*} M}(f):=\varsigma^{M}\left(\tau_{d} \circ G^{\prime} f \circ N_{c}\right)$. The pullback extends to module morphisms and is functorial.

Given two languages over different object types $T$ and $T^{\prime}$, modelled as relative monads $P$ and $Q$ on $\Delta^{T}$ and $\Delta^{T^{\prime}}$, respectively, we model a translation from $P$ to $Q$ by a colax monad morphism whose underlying functors are retyping functors:

Definition 8 (Retyping Functor). Let $g: T \rightarrow T^{\prime}$ be a map of sets, and let $\mathcal{C}$ be a cocomplete category. The map $g$ induces a functor $g^{*}: \mathcal{C}^{T^{\prime}} \rightarrow \mathcal{C}^{T}$ by postcomposition, $W \mapsto W \circ g$. The retyping functor $\vec{g}: \mathcal{C}^{T} \rightarrow \mathcal{C}^{T^{\prime}}$ associated to $g: T \rightarrow T^{\prime}$ is defined as the left Kan extension operation along $g$, that is, we have an adjunction $\vec{g} \dashv g^{*}$.

Remark 9. We are going to use the following instance of Def. 6: $P$ and $Q$ are monads - e.g., languages - on $\Delta^{T}$ and $\Delta^{T^{\prime}}$, for sets $T$ and $T^{\prime}$ of object types. The functors $G$ and $G^{\prime}$ are the retyping functors (cf. Def. 8) associated to some translation of types $g: T \rightarrow T^{\prime}$, and $N$ is the identity transformation. Then $\tau$ denotes a translation of terms from $P$ to $Q$ :
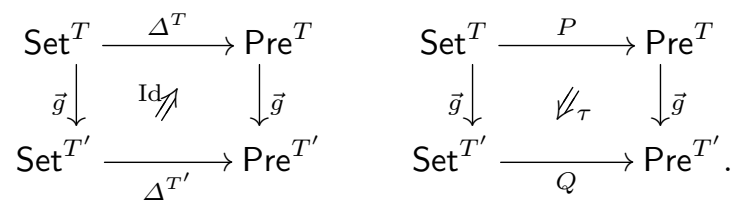
A family of constructors, such as the family $\left(\mathrm{Abs}_{s, t}\right)_{s, t \in T_{\mathrm{TLC}}}$ of Ex. 5, is modelled via a family of module morphisms of suitable domain and codomain. Equivalently, via uncurrying, we can consider such a family as one module morphism between two suitable modules: intuitively, the idea is to write $\operatorname{Abs}(V, s, t): \operatorname{TLC} \beta\left(V^{s}\right)(t) \rightarrow$ $\operatorname{TLC} \beta(V)(s \rightsquigarrow t)$ instead of $\operatorname{Abs}_{s, t}(V)$. For this to work, an object of the domain category of the source and target modules of Abs must be of the form $(V, s, t)$, where $V$ is a context and $s, t \in T_{\mathrm{TLC}}$. More generally:

Definition 10 (Pointed index sets). Given a category $\mathcal{C}$, a set $T$ and a natural number $n$, we denote by $\mathcal{C}_{n}^{T}$ the category with, as objects, diagrams of the form $n \stackrel{\mathbf{t}}{\rightarrow} T \stackrel{V}{\rightarrow} \mathcal{C}$, written $\left(V, t_{1}, \ldots, t_{n}\right)$ with $t_{i}:=\mathbf{t}(i)$. A morphism $h$ to another such $(W, \mathbf{t})$ with the same pointing map $\mathbf{t}$ is given by a morphism $h: V \rightarrow W$ in $\mathcal{C}^{T}$.

Any functor $F: \mathcal{C}^{T} \rightarrow \mathcal{D}^{T}$ extends to $F_{n}: \mathcal{C}_{n}^{T} \rightarrow \mathcal{D}_{n}^{T}$ via $F_{n}(V, \mathbf{t}):=(F V, \mathbf{t})$. Also, any relative monad $R$ over $F$ induces a monad $R_{n}$ over $F_{n}$.

Given a map of sets $g: T \rightarrow T^{\prime}$, by postcomposing the pointing map with $g$, the retyping functor ( $c f$. Def. 8) generalizes to the functor

$$
\vec{g}(n): \mathcal{C}_{n}^{T} \rightarrow \mathcal{C}_{n}^{T^{\prime}}, \quad(V, \mathbf{t}) \mapsto(\vec{g} V, g \circ \mathbf{t}) .
$$

The category $\mathcal{C}_{n}^{T}$ consists of $T^{n}$ copies of $\mathcal{C}^{T}$, which do not interact. Due to the "markers" $\left(t_{1}, \ldots, t_{n}\right)$ we can act differently on each copy, cf. Defs. 12 and 13.

Two important constructions on modules over monads of [2], derivation and fibre modules, carry over to modules over monads on $\Delta^{T}$. Intuitively, derivation corresponds to considering terms in an extended context, whereas the fibre corresponds to picking terms of a specific object type. Since we consider varying sets of types, the object type for context extension and fibre is chosen through a natural transformation, which picks an element of any set.

Given $u \in T$, we denote by $D(u) \in \operatorname{Set}^{T}$ the context with $D(u)(u)=\{*\}$ and $D(u)(t)=\emptyset$ for $u \neq t$. For a context $V \in \operatorname{Set}^{T}$ we set $V^{* u}:=V+D(u)$.

Given a category $\mathcal{C}$ and $n \in \mathbb{N}$, we denote by $\mathcal{T C}_{n}$ the category an object of which is a triple $(T, V, \mathbf{t})$ of a set $T$, a $T$-indexed family $V$ of objects of $\mathcal{C}$ and a vector $\mathbf{t}$ of length $n$ of elements of $T$. Note that for a fixed set $T$, the category $\mathcal{C}_{n}^{T}$ is the fibre over $T$ of the forgetful functor $\mathcal{T} U_{n}: \mathcal{T} \mathcal{C}_{n} \rightarrow$ Set which maps an object $(T, V, \mathbf{t})$ to its indexing set $T$. Let $1: \mathcal{T C}_{n} \rightarrow$ Set be the constant functor mapping to the singleton set. For a natural transformation $\tau: 1 \Rightarrow \mathcal{T} U_{n}$, we write $\tau(T, V, \mathbf{t}):=\tau(T, V, \mathbf{t})(*) \in T$, i.e. we omit the argument from the singleton set. Intuitively, such $\tau$ picks an element of $T$ of any object $(T, V, \mathbf{t}) \in \mathcal{T C}_{n}$.

Example 11. For $1 \leq k \leq n$, we denote by $k: 1 \Rightarrow \mathcal{T} U_{n}: \mathcal{T C}{ }_{n} \rightarrow$ Set the natural transformation such that $k(T, V, \mathbf{t})(*):=\mathbf{t}(k)$.

Definition 12 (Context Extension). Let $\tau$ be as above, and let $T$ be a fixed set. Given a monad $P$ on $\Delta_{n}^{T}$ and a $P$-module $M$ with codomain $\mathcal{E}$, we define the derived module of $M$ with respect to $\tau$ by setting $M^{\tau}(V, \mathbf{t}):=M\left(V^{* \tau(T, V, \mathbf{t})}, \mathbf{t}\right)$. 
Definition 13 (Fibre). Let $\tau$ be as in Def. 12. Let $P$ be a monad on $\Delta_{n}^{T}$ and $M$ be a $P$-module with codomain category $\mathcal{E}_{n}^{T}$. The fibre module $[M]_{\tau}$ of $M$ with respect to $\tau$ has object map $(V, \mathbf{t}) \mapsto M(V, \mathbf{t})(\tau(T, V, \mathbf{t}))$, that is, the component $\tau(T, V, \mathbf{t})$ of $M$, forgetting also the pointing map $\mathbf{t}$.

Example 14 (Ex. 5 cont.). Let $T:=T_{\mathrm{TLC}}$. According to Def. 10, we have a relative monad - and its associated tautological module - TLC $\beta_{2}$ on the functor $\Delta_{2}^{T}: \operatorname{Set}_{2}^{T} \rightarrow \operatorname{Pre}_{2}^{T}$. Let $i: 1 \Rightarrow T U_{2}: T \mathcal{C}_{2} \rightarrow$ Set, for $i=1,2$, be a natural transformation as in Ex. 11. Then we have a $\operatorname{TLC} \beta_{2}-$ module

$$
\operatorname{TLC} \beta_{2}{ }^{1}:(V, s, t) \mapsto \operatorname{TLC} \beta_{2}\left(V^{s}, s, t\right) .
$$

We also have a TLC $\beta_{2}$-module

$$
\left[\mathrm{TLC} \beta_{2}\right]_{2}:(V, s, t) \mapsto \operatorname{TLC} \beta_{2}(V, s, t)(t) .
$$

Again, as in Ex. 5, analogous remarks hold for TLC $\Delta$.

Remark 15 (Module of Higher Degree corresponds to a Family of Modules). Let $T$ be a set and let $R$ be a monad on the functor $\Delta^{T}$. Then a module $M$ over the monad $R_{n}$ corresponds precisely to a family of $R$-modules $\left(M_{\mathbf{t}}\right)_{\mathbf{t} \in T^{n}}$ by (un)currying. Similarly, a morphism $\alpha: M \rightarrow N$ of modules of degree $n$ is equivalent to a family $\left(\alpha_{\mathbf{t}}\right)_{\mathbf{t} \in T^{n}}$ of morphisms of modules of degree zero with $\alpha_{\mathbf{t}}: M_{\mathbf{t}} \rightarrow N_{\mathbf{t}}$.

\section{Signatures, Representations, Initiality}

We combine the techniques of [2] and [1] in order to obtain an initiality result for simple type systems with reductions on the term level. As an example, we specify, via the iteration principle stemming from the universal property, a semantically faithful translation from PCF with its usual reduction relation to the untyped lambda calculus with beta reduction. Analogously to [1], we define a notion of 2-signature with two levels: a syntactic level specifying types and terms of a language, and, on top, a semantic level specifying reduction rules on the terms.

The syntactic level itself - given by a 1-signature $(S, \Sigma)$, cf. Def. $29-$ specifies the types of the language, via an algebraic signature $S$, as well as terms that are typed over the types specified by $S$, via a signature $\Sigma$ over $S$. In a first result (cf. Lem. 34) we characterize the language generated by a 1-signature, and equipped with the equality preorder, as an initial object of a category of representations. An instance of this theorem is given in Ex. 22, where TLC $\Delta$, equipped with two module morphisms given by the constructors Abs and App, is characterized as the initial representation of a suitable 1-signature.

Afterwards we equip 1-signatures with inequations, yielding 2-signatures (cf. Def. 42). We prove an initiality result for those 2-signatures (cf. Thm. 44), an instance of which characterizes the simply-typed lambda calculus TLC $\beta$ with beta reduction as initial representation (cf. Ex. 43). 
Signatures for Types We consider sets of types that are specified by algebraic signatures, which are presented in [2]. We review briefly:

Definition 16 (Algebraic Signature). An algebraic signature is given by a family of natural numbers.

Intuitively, each natural number of such a family specifies the number of arguments of its associated type constructor.

Example 17. The types of the simply-typed lambda calculus are specified via the algebraic signature $S_{\mathrm{TLC}}:=\{*: 0,(\rightsquigarrow): 2\}$.

Example 18. The language PCF [14] is a simply-typed lambda calculus with a fixed point operator and arithmetic constants. The signature of the types of PCF is given by $S_{\mathrm{PCF}}:=\{\iota: 0, o: 0,(\Rightarrow): 2\}$. A representation $T$ of $S_{\mathrm{PCF}}$ is given by a set $T$ and three operations of suitable arities. A morphism of representations is a map of sets compatible with the operations on either side.

Lemma 19. Let $S$ be an algebraic signature. The category of representations of $S$ has an initial object $\hat{S}$.

Signatures for Terms For the rest of the section, let $S$ be a signature for types. Signatures for terms over $S$ are syntactically defined as in [2]. We call degree of an arity the number of object type variables appearing in the arity. For instance, the signature $\Sigma_{\mathrm{TLC}}$ of simply-typed lambda terms over the signature $S_{\mathrm{TLC}}$ (cf. Ex. 17) is given by two arities of degree 2 :

$$
\Sigma_{\mathrm{TLC}}:=\{\operatorname{abs}:[([1], 2)] \rightarrow(1 \rightsquigarrow 2), \quad \text { app }:[([], 1 \rightsquigarrow 2),([], 1)] \rightarrow 2\} .
$$

Intuitively, the numbers vary over object types. More precisely, for any representation of $S_{\text {TLC }}$ in a set $T$, the numbers vary over elements of $T$.

In order to define representations of such a signature $(S, \Sigma)$, we need to consider set families where the indexing set is equipped with a representation of the type signature $S$ :

Definition 20. Given a category $\mathcal{C}-$ e.g., the category Set of sets - we define the category $S \mathcal{C}_{n}$ to be the category an object of which is a triple $(T, V, \mathbf{t})$ where $T$ is a representation of $S$, the object $V \in \mathcal{C}^{T}$ is a $T$-indexed family of objects of $\mathcal{C}$ and $\mathbf{t}$ is a vector of elements of $T$ of length $n$. We denote by $S U_{n}: S \mathcal{C}_{n} \rightarrow$ Set the functor mapping an object $(T, V, \mathbf{t})$ to the underlying set $T$.

We have a forgetful functor $S \mathcal{C}_{n} \rightarrow \mathcal{T C}_{n}$ which forgets the representation structure. On the other hand, any representation $T$ of $S$ in a set $T$ gives rise to a functor $\mathcal{C}_{n}^{T} \rightarrow S \mathcal{C}_{n}$, which "attaches" the representation structure.

Recall from [2] that $S(n)$ denotes terms of $S$ with free variables in $\{1, \ldots, n\}$. The meaning of a term $s \in S(n)$ as a natural transformation $s: 1 \Rightarrow S U_{n}$ : $S \mathcal{C}_{n} \rightarrow$ Set is given by recursion on the structure of $s:$ 
Definition 21 (Canonical Natural Transformation). Let $s \in S(n)$ be a type of degree $n$. Then $s$ denotes a natural transformation $s: 1 \Rightarrow S U_{n}: S \mathcal{C}_{n} \rightarrow$ Set defined recursively on the structure of $s$ as follows: for $s=\alpha\left(a_{1}, \ldots, a_{k}\right)$ the image of a constructor $\alpha \in S$ we set

$$
s(T, V, \mathbf{t})=\alpha\left(a_{1}(T, V, \mathbf{t}), \ldots, a_{k}(T, V, \mathbf{t})\right)
$$

and for $s=m$ with $1 \leq m \leq n$ we define $s(T, V, \mathbf{t})=\mathbf{t}(m)$. We call a natural transformation of the form $s \in S(n)$ canonical.

The natural transformations of Ex. 11 yield examples of canonical transformations. We now define representations of the 1-signature $\left(S_{\mathrm{TLC}}, \Sigma_{\mathrm{TLC}}\right)$ of the simplytyped lambda calculus. Afterwards we define general 1-signatures and their representations.

Example 22 (Ex. 14 cont.). Let $S:=S_{\text {TLC }}$ be the signature for types of TLC as in Ex. 17. We denote by $i: 1 \Rightarrow S U_{2}: S C_{2} \rightarrow$ Set, for $i=1,2$, the natural transformations defined analogously to those of Ex. 14. We define the transformation $1 \rightsquigarrow 2: 1 \Rightarrow S U_{2}$ as

$$
(1 \rightsquigarrow 2)(V, s, t)(*):=s \rightsquigarrow t .
$$

The constructors of the simply-typed lambda calculus thus constitute the carriers of two module morphisms,

$$
\begin{aligned}
& \text { Abs : }\left[\operatorname{TLC}_{2}{ }^{1}\right]_{2} \rightarrow\left[\mathrm{TLC} \Delta_{2}\right]_{1 \rightsquigarrow 2} \\
& \text { App : }\left[\mathrm{TLC} \Delta_{2}\right]_{1 \rightsquigarrow 2} \times\left[\mathrm{TLC} \Delta_{2}\right]_{1} \rightarrow\left[\mathrm{TLC} \Delta_{2}\right]_{2} .
\end{aligned}
$$

Altogether we model the simply-typed lambda calculus with equality relation via the following categorical structure:

- the relative monad TLC $\Delta$ on $\Delta^{T \text { TLC }}$ and

- two morphisms of TLC $\Delta_{2}$-modules Abs and App of type as in Disp. (2).

We thus define a representation of the simply-typed lambda calculus, specified by the signature $\left(S_{\mathrm{TLC}}, \Sigma_{\mathrm{TLC}}\right)$ (cf. Disp. (1)), as a representation $T$ of $S_{\mathrm{TLC}}$ in a set $T$, a monad $P$ on $\Delta^{T}$ and two morphisms of $P_{2}$-modules

$$
\text { Abs : }\left[P_{2}{ }^{1}\right]_{2} \rightarrow\left[P_{2}\right]_{1 \rightsquigarrow 2} \text { and App }:\left[P_{2}\right]_{1 \rightsquigarrow 2} \times\left[P_{2}\right]_{1} \rightarrow\left[P_{2}\right]_{2} .
$$

Together with a suitable definition of morphisms of representations, this yields a category in which the triple (TLC $\Delta, \mathrm{Abs}, \mathrm{App})$ is the initial object.

In general, an arity over $S$ of degree $n \in \mathbb{N}$ is given by a pair of functors, each of which associates, to any suitable monad $P$, a source $\operatorname{dom}(s, P)$ and a target $\operatorname{dom}(s, P)$ of a $P_{n}$-module morphism. Each such functor is called a half-arity. Representing an arity in the monad $P$ then means specifying a module morphism $\operatorname{dom}(s, P) \rightarrow \operatorname{cod}(s, P)$.

We define the source and target categories of half-arities; an object of the source category is a pair of a representation of $S$ in a set $T$ and a monad on $\Delta^{T}$. 
Definition 23 (Relative $S$-Monad). The category $S$-RMnd of relative $S$ monads is the category whose objects are pairs $(T, P)$ of a representation $T$ of $S$ and a relative monad $P$ on $\Delta^{T}$. A morphism from $(T, P)$ to $\left(T^{\prime}, P^{\prime}\right)$ is a pair $(g, f)$ of a morphism of $S$-representations $g: T \rightarrow T^{\prime}$ and a morphism of relative monads $f: P \rightarrow P^{\prime}$ over $\vec{g}$ as in Rem. 9 .

Given $n \in \mathbb{N}$, we write $S-\mathrm{RMnd}_{n}$ for the category whose objects are pairs $(T, P)$ of a representation $T$ of $S$ and a relative monad $P$ over $\Delta_{n}^{T}$. A morphism from $(T, P)$ to $\left(T^{\prime}, P^{\prime}\right)$ is a pair $(g, f)$ of a morphism of $S$-representations $g: T \rightarrow T^{\prime}$ and a monad morphism $f: P \rightarrow P^{\prime}$ over the retyping functor $\vec{g}(n)$ (Def. 10).

The target categories mix modules over different relative monads:

Definition 24. Given $n \in \mathbb{N}$, an algebraic signature $S$ and a category $\mathcal{D}$, we call $\operatorname{LRMod}_{n}(S, \mathcal{D})$ the category an object of which is a pair $(P, M)$ of a relative $S$ monad $P \in S-\mathrm{RMnd}_{n}$ and a $P$-module with codomain $\mathcal{D}$. A morphism to another such $(Q, N)$ is a pair $(f, h)$ of a morphism of relative $S$-monads $f: P \rightarrow Q$ in $S-\mathrm{RMnd}_{n}$ and a morphism of relative modules $h: M \rightarrow f^{*} N$.

We sometimes just write the module - i.e. the second - component of an object or morphism of the large category of modules. Given $M \in \operatorname{LRMod}_{n}(S, \mathcal{D})$, we thus write $M(V, \mathbf{t})$ or $M_{V, \mathbf{t}}$ for the value of the module on the object $(V, \mathbf{t})$.

A half-arity over $S$ of degree $n$ is a functor from relative $S$-monads to the category of large modules of degree $n$.

Definition 25. Given an algebraic signature $S$ and $n \in \mathbb{N}$, $a$ half-arity over $S$ of degree $n$ is a functor $\alpha: S$-RMnd $\rightarrow \operatorname{LRMod}_{n}(S$, Pre $)$ which is pre-inverse to the forgetful functor.

The basic building brick for the half-arities we consider is the tautological module:

Definition 26. To any relative $S-$ monad $R$ we associate the tautological module of $R_{n}$ (cf. Def. 10),

$$
\Theta_{n}(R):=\left(R_{n}, R_{n}\right) \in \operatorname{LRMod}_{n}\left(S, \operatorname{Pre}_{n}^{T}\right) .
$$

From the tautological module, we build classic half-arities using canonical natural transformations (cf. Def. 21); these transformations specify context extension (derivation) and selection of specific object types (fibre):

Definition 27 (Classic Half-Arity). The following clauses define an inductive set of classic half-arities, to which we restrict our attention:

- The constant functor $*: R \mapsto 1_{\mathrm{RMod}(R, \mathrm{Pre})}$ is a classic half-arity.

- Given any canonical natural transformation $\tau: 1 \Rightarrow S U_{n}$ (cf. Def. 21), the point-wise fibre module with respect to $\tau$ (cf. Def. 13) of the tautological module $\Theta_{n}: R \mapsto\left(R_{n}, R_{n}\right)$ (cf. Def. 26) is a classic half-arity of degree $n$,

$$
\left[\Theta_{n}\right]_{\tau}: S \text {-RMnd } \rightarrow \operatorname{LRMod}_{n}(S, \text { Pre }), \quad R \mapsto\left[R_{n}\right]_{\tau} .
$$


- Given any (classic) half-arity $M: S$-Mnd $\rightarrow \operatorname{LMod}_{n}(S$, Pre) of degree $n$ and a canonical natural transformation $\tau: 1 \Rightarrow S U_{n}$, the point-wise derivation of $M$ with respect to $\tau$ is a (classic) half-arity of degree $n$,

$$
M^{\tau}: S \text {-RMnd } \rightarrow \operatorname{LRMod}_{n}(S, \text { Pre }), \quad R \mapsto(M(R))^{\tau} .
$$

- Given two (classic) half-arities $M$ and $N$ of degree $n$, their pointwise product of modules $M \times N$ is again a (classic) half-arity of degree $n$.

A half-arity of degree $n$ thus associates, to any relative $S$-monad $P$ over a set of types $T$, a family of $P$-modules indexed by $T^{n}$, cf. Rem. 15 .

An arity of degree $n \in \mathbb{N}$ for terms over an algebraic signature $S$ is defined to be a pair of functors from relative $S$-monads to modules in $\operatorname{LRMod}_{n}(S$, Pre). The degree $n$ corresponds to the number of object type indices of its associated constructor. For instance, the arities of Abs and App of Disp. (1) are of degree 2.

Definition 28 (Term-Arity, Signature over $S$ ). $A$ classic arity $\alpha$ over $S$ of degree $n$ is a pair $s=(\operatorname{dom}(\alpha), \operatorname{cod}(\alpha))$ of half-arities over $S$ of degree $n$ such that $\operatorname{dom}(\alpha)$ is classic and $\operatorname{cod}(\alpha)$ is of the form $\left[\Theta_{n}\right]_{\tau}$ for some canonical transformation $\tau$ as in Def. 21. We write $\operatorname{dom}(\alpha) \rightarrow \operatorname{cod}(\alpha)$ for the arity $\alpha$, and $\operatorname{dom}(\alpha, R):=\operatorname{dom}(\alpha)(R)$ and similar for the codomain and morphisms of relative $S$-monads. A term-signature $\Sigma$ over $S$ is a family of classic arities (of varying degree) over $S$.

Definition 29 (1-Signature). A 1-signature is a pair $(S, \Sigma)$ consisting of an algebraic signature $S$ for sorts and a term-signature $\Sigma$ over $S$.

Example 30 (Ex. 22 cont.). The terms of the simply typed lambda calculus over the type signature of Ex. 17 are given by the arities

$$
\text { abs : }[\Theta]_{2}^{1} \rightarrow[\Theta]_{1 \rightsquigarrow 2}, \quad \text { app : }[\Theta]_{1 \rightsquigarrow 2} \times[\Theta]_{1} \rightarrow[\Theta]_{2},
$$

both of which are of degree 2 - we leave the degree implicit. The outer lower index and the exponent are to be interpreted as de Bruijn variables, ranging over types. They indicate the fibre (cf. Def. 13) and derivation (cf. Def. 12), respectively, in the special case where the corresponding natural transformation is given by a natural number as in Def. 21 .

Example 31 (Ex. 18 cont.). The term-signature of PCF consists of an arity for abstraction and an arity for application, each of degree 2, an arity (of degree 1) for the fixed point operator, and one arity of degree 0 for each logic and arithmetic constant - some of which we omit:

$$
\begin{gathered}
\text { abs : }[\Theta]_{2}^{1} \rightarrow[\Theta]_{1 \Rightarrow 2}, \quad \text { app }:[\Theta]_{1 \Rightarrow 2} \times[\Theta]_{1} \rightarrow[\Theta]_{2}, \quad \text { Fix : }[\Theta]_{1 \Rightarrow 1} \rightarrow[\Theta]_{1}, \\
\mathbf{n}: * \rightarrow[\Theta]_{\iota} \text { for } n \in \mathbb{N}, \quad \text { Succ }: * \rightarrow[\Theta]_{\iota \Rightarrow \iota}, \quad \text { Zero? : } * \rightarrow[\Theta]_{\iota \Rightarrow o}
\end{gathered}
$$

Definition 32 (Representation of an Arity, of a 1-Signature over $S$ ). A representation $r$ of an arity $\alpha$ over $S$ in an $S$-monad $R$ is a morphism of relative modules $r: \operatorname{dom}(\alpha, R) \rightarrow \operatorname{cod}(\alpha, R)$. A representation $R$ of a signature over $S$ is a given by a relative $S$-monad - called $R$ as well - and a representation $\alpha^{R}$ of each arity $\alpha$ of $S$ in $R$. 
Representations of $(S, \Sigma)$ are the objects of a category $\operatorname{Rep}^{\Delta}(S, \Sigma)$, whose morphisms are defined as follows:

Definition 33 (Morphism of Representations). Given representations $P$ and $R$ of a typed signature $(S, \Sigma)$, a morphism of representations $f: P \rightarrow R$ is given by a morphism of relative $S$-monads $f: P \rightarrow R$, such that for any arity $\alpha$ of $\Sigma$ the following diagram of module morphisms commutes:

$$
\operatorname{cod}(\alpha, f) \circ \alpha^{P}=\alpha^{R} \circ \operatorname{dom}(\alpha, f) .
$$

Lemma 34. For any 1-signature $(S, \Sigma)$, the category of representations of $(S, \Sigma)$ has an initial object.

Proof. The initial object is obtained, analogously to the untyped case (cf. [1]), via an adjunction $\Delta_{*} \dashv U_{*}$ between the categories of representations of $(S, \Sigma)$ in relative monads and those in monads as in [2]. We use that left adjoints are cocontinuous, and thus preserve initial objects.

Inequations \& 2-Signatures An inequation associates, to any representation of $(S, \Sigma)$ in a relative monad $P$, two parallel morphisms of $P$-modules. Similarly to arities, an inequation (of higher degree) may be given by a family of inequations, indexed by object types. Consider the simply-typed lambda calculus, which was defined with typed abstraction and application. Similarly, we have a typed substitution operation for TLC and, more generally, for any monad on $\Delta^{T}$ (cf. Def. 36). For $s, t \in T_{\mathrm{TLC}}$ and $M \in \operatorname{TLC}\left(V^{* s}\right)_{t}$ and $N \in \operatorname{TLC}(V)_{s}$, beta reduction is specified by

$$
\lambda M(N) \rightsquigarrow M[*:=N],
$$

where our notation hides the fact that abstraction, application and substitution are typed operations. More formally, such a reduction rule might read as a family of inequations between morphisms of modules

$$
\left.\operatorname{app}_{s, t} \circ\left(\operatorname{abs}_{s, t} \times \mathrm{id}\right) \leq{ }_{-}\left[*^{s}:=_{t}\right]\right],
$$

where $s, t \in T_{\mathrm{TLC}}$ range over types of the simply-typed lambda calculus. Analogously to 1-signatures, we want to specify the beta rule without referring to the set $T_{\mathrm{TLC}}$, but instead express it for an arbitrary representation $R$ of the typed signature $\left(S_{\mathrm{TLC}}, \Sigma_{\mathrm{TLC}}\right)$ (cf. Ex. 30$)$, as in

$$
\operatorname{app}^{R} \circ\left(\operatorname{abs}^{R} \times \mathrm{id}\right) \leq{ }_{-}\left[*:=_{-}\right],
$$

where both the left and the right side of the inequation are given by suitable $R$-module morphisms of degree 2 .

Definition 35. Let $(S, \Sigma)$ be a 1-signature, and let $U: \operatorname{Rep}^{\Delta}(S, \Sigma) \rightarrow S$-RMnd be the forgetful functor. Given two (classic) half-arities $\operatorname{dom}(s)$ and $\operatorname{cod}(s)$ of degree $n \in \mathbb{N}$, a half-equation $\alpha: \operatorname{dom}(s) \rightarrow \operatorname{cod}(s)$ of degree $n$ over $(S, \Sigma)$ is a natural transformation $\alpha: \operatorname{dom}(s) \circ U \rightarrow \operatorname{cod}(s) \circ U$. We call an inequation classic when its codomain is given by a classic half-arity. 
Definition 36 (Substitution of one Variable as a Half-Equation). Let T be a (nonempty) set and let $P$ be a monad over $\Delta^{T}$. For any $s, t \in T$ and $X \in \operatorname{Set}^{T}$ we define a binary substitution operation $(y, z) \mapsto y[*:=z]:=\sigma\left(\left[\eta_{X}, x \mapsto z\right]\right)(y)$. For any pair $(s, t) \in T^{2}$, we thus obtain a morphism of $P$-modules

$$
\operatorname{subst}_{s, t}^{P}:\left[P^{s}\right]_{t} \times[P]_{s} \rightarrow[P]_{t} .
$$

By Rem. 15 this family is equivalent to a module morphism of degree 2. We thus have a half-equation of degree 2 with classic domain and codomain over any typed signature,

$$
\text { subst : } R \mapsto \operatorname{subst}^{R}:\left[R_{2}^{1}\right]_{2} \times\left[R_{2}\right]_{1} \rightarrow\left[R_{2}\right]_{2} .
$$

Example 37 (Ex. 30 cont.). The following map yields a half-equation over the signature TLC, as well as over the signature of PCF:

$$
\operatorname{app} \circ(\operatorname{abs} \times \mathrm{id}): R \mapsto \operatorname{app}^{R} \circ\left(\operatorname{abs}^{R} \times \mathrm{id}^{R}\right):\left[R_{2}^{1}\right]_{2} \times\left[R_{2}\right]_{1} \rightarrow\left[R_{2}\right]_{2} .
$$

Definition 38 (Inequation). Given a signature $(S, \Sigma)$, an inequation over $(S, \Sigma)$, or $(S, \Sigma)$-inequation, of degree $n \in \mathbb{N}$ is a pair of parallel half-equations of degree $n$. We write $\alpha \leq \gamma$ for the inequation $(\alpha, \gamma)$.

Example 39 (Beta Reduction). For any suitable 1-signature - i.e. for any 1signature that has an arity for abstraction and an arity for application - we specify beta reduction using the parallel half-equations of Def. 36 and Ex. 37:

$$
\text { app } \circ(\text { abs } \times \text { id }) \leq \text { subst : }\left[\Theta_{2}\right]_{2}^{1} \times\left[\Theta_{2}\right]_{1} \rightarrow\left[\Theta_{2}\right]_{2} .
$$

Example 40 (Fixpoints and Arithmetics of PCF). We specify some of the reduction rules of PCF via inequations over the 1-signature of PCF (cf. Ex. 31); for space reasons we refrain from specifying all of them. The reader may fill in the missing inequations, whose informal specification can be found, e.g., in [13].

$$
\begin{aligned}
\text { Fix } & \leq \text { app } \circ(\text { id, Fix }):[\Theta]_{1 \Rightarrow 1} \rightarrow[\Theta]_{1} \\
\text { app } \circ(\text { Pred, } 0) & \leq \mathbf{0}: * \rightarrow[\Theta]_{\iota} \\
\text { app } \circ(\text { Pred, app } \circ(\text { Succ, }, \mathbf{n})) & \leq \mathbf{n}: * \rightarrow[\Theta]_{\iota} \\
\operatorname{app} \circ(\text { Zero?, } \mathbf{0}) & \leq \mathbf{T}: * \rightarrow[\Theta]_{o} \\
\text { app } \circ(\text { Zero?, app } \circ(\text { Succ, }, \mathbf{n})) & \leq \mathbf{F}: * \rightarrow[\Theta]_{o}
\end{aligned}
$$

Definition 41 (Representation of Inequations). $A$ representation of an $(S, \Sigma)$-inequation $\alpha \leq \gamma: U \rightarrow V$ (of degree $n$ ) is any representation $R$ over a set of types $T$ of $(S, \Sigma)$ such that $\alpha^{R} \leq \gamma^{R}$ pointwise, i.e. if for any pointed context $(X, \mathbf{t}) \in \operatorname{Set}^{T} \times T^{n}$, any $t \in T$ and any $y \in U_{(X, \mathbf{t})}^{R}(t), \alpha^{R}(y) \leq \gamma^{R}(y)$, where we omit the sort argument $t$ as well as the context $(X, \mathbf{t})$ from $\alpha$ and $\gamma$. We say that such a representation $R$ satisfies the inequation $\alpha \leq \gamma$.

The category of representations of $((S, \Sigma), A)$ is defined as the full subcategory of $\operatorname{Rep}^{\Delta}(S, \Sigma)$ of representations satisfying each inequation of A. According to Rem. 15, the above inequation is equivalent to ask whether, for any $\mathbf{t} \in T^{n}$, any $t \in T$ and any $y \in U_{\mathbf{t}}^{R}(X)(t), \alpha_{\mathbf{t}}^{R}(y) \leq \gamma_{\mathbf{t}}^{R}(y)$. 
Definition 42 (2-Signature). A 2-signature is a pair given by a 1-signature $(S, \Sigma)$ and a set $A$ of classic inequations over $(S, \Sigma)$.

Example 43 (Representations of TLC with $\beta$ ). A representation of $\left(S_{\mathrm{TLC}}, \Sigma_{\mathrm{TLC}}, \beta\right)$ is given by a representation ( $P, \mathrm{Abs}, \mathrm{App})$ of $\left(S_{\mathrm{TLC}}, \Sigma_{\mathrm{TLC}}\right)$ over a set $T$ "of types" such that, for any context $V \in \operatorname{Set}^{T}$, any $s, t \in T$ and any $M \in P^{s}(V)(t)$ and $N \in P(V)(s)$,

$$
\operatorname{App}_{s, t}\left(\operatorname{Abs}_{s, t}(M), N\right) \leq M[*:=N] .
$$

The initial such representation is given by the triple ( $T L C \beta, A b s, A p p)$, where

$$
\begin{aligned}
& \text { Abs : }\left[\mathrm{TLC} \beta_{2}{ }^{1}\right]_{2} \rightarrow\left[\mathrm{TLC} \beta_{2}\right]_{1 \rightsquigarrow 2} \\
& \text { App : }\left[\mathrm{TLC} \beta_{2}\right]_{1 \rightsquigarrow 2} \times\left[\mathrm{TLC} \beta_{2}\right]_{1} \rightarrow\left[\mathrm{TLC} \beta_{2}\right]_{2} .
\end{aligned}
$$

The above example is an instance of the following general theorem for 2-signatures:

Theorem 44. For any set of classic $(S, \Sigma)$-inequations $A$, the category of representations of $((S, \Sigma), A)$ has an initial object.

A proof of the theorem can be found in the author's $\mathrm{PhD}$ thesis [3].

The following remark gives a "manual" on how to use the universal property of initiality in order to specify a translation between two languages:

Remark 45 (Iteration Principle by Initiality). The universal property of the language generated by a 2-signature yields an iteration principle to define maps - translations - on this language, which are compatible by construction with substitution and reduction in the source and target languages. A translation from the language generated by $(S, \Sigma, A)$ to the language generated by $\left(S^{\prime}, \Sigma^{\prime}, A^{\prime}\right)$ can be obtained, via the universal property, as an initial morphism in $\operatorname{Rep}^{\Delta}(S, \Sigma, A)$, obtained by equipping the relative monad $\hat{\Sigma}_{A^{\prime}}^{\prime}$ underlying the target language with a representation of the signature $(S, \Sigma, A)$. In more detail:

1. we give a representation of the type signature $S$ in the set $\hat{S}^{\prime}$. By initiality of $\hat{S}$, this yields a translation $\hat{S} \rightarrow \hat{S}^{\prime}$ of sorts.

2. Afterwards, we specify a representation of the term signature $\Sigma$ in the monad $\hat{\Sigma}_{A^{\prime}}^{\prime}$ by defining suitable (families) of morphisms of $\hat{\Sigma}_{A^{\prime}}^{\prime}$-modules. This yields a representation $R$ of $(S, \Sigma)$ in the monad $\hat{\Sigma}_{A^{\prime}}^{\prime}$.

3. Finally, we verify that the representation $R$ of $(S, \Sigma)$ satisfies the inequations of $A$, that is, we check whether, for each $\alpha \leq \gamma: \operatorname{dom}(\alpha) \rightarrow \operatorname{cod}(\alpha) \in A$, and for each context $V$, each $t \in \hat{S}$ and $x \in \operatorname{dom}(\alpha)_{V}^{R}(t), \alpha^{R}(x) \leq \gamma^{R}(x)$.

Example 46 (Translation from PCF to ULC). We use the aforementioned iteration principle to specify a translation from PCF to ULC, which is semantically faithful with respect to the usual reduction relation of $\mathrm{PCF}$ - generated by the inequations of Ex. 40 (and some more, see [14]) - and beta reduction of ULC. For space reasons, we cannot present this example here; we refer to [3]. This example initiality of the types and terms of PCF with its reductions, and a translation to ULC with beta reduction via associated category-theoretic iteration operator - has also been implemented in the proof assistant Coq. The source files and documentation are available on http://math.unice.fr/laboratoire/logiciels. 


\section{References}

1. Ahrens, B.: Modules over relative monads for syntax and semantics (2011), http: //arxiv.org/abs/1107.5252, to be published in Math. Struct. in Comp. Science

2. Ahrens, B.: Extended Initiality for Typed Abstract Syntax. Logical Methods in Computer Science 8(2), 1 - 35 (2012)

3. Ahrens, B.: Initiality for Typed Syntax and Semantics. Ph.D. thesis, University of Nice Sophia Antipolis, France (2012), http://benedikt-ahrens.org

4. Altenkirch, T., Chapman, J., Uustalu, T.: Monads Need Not Be Endofunctors. In: Ong, C.H.L. (ed.) FOSSACS. Lecture Notes in Computer Science, vol. 6014, pp. 297-311. Springer (2010)

5. Coq: The Coq Proof Assistant (2010), http://coq.inria.fr

6. Fernández, M., Gabbay, M.J.: Nominal rewriting. Information and Computation 205(6), 917-965 (6 2007)

7. Fiore, M.P., Hur, C.K.: Equational systems and free constructions (extended abstract). In: Arge, L., Cachin, C., Jurdzinski, T., Tarlecki, A. (eds.) ICALP. Lecture Notes in Computer Science, vol. 4596, pp. 607-618. Springer (2007)

8. Ghani, N., Lüth, C.: Rewriting via coinserters. Nord. J. Comput. 10(4), 290-312 (2003)

9. Hirschowitz, A., Maggesi, M.: Modules over monads and linearity. In: Leivant, D., de Queiroz, R.J.G.B. (eds.) WoLLIC. Lecture Notes in Computer Science, vol. 4576, pp. 218-237. Springer (2007)

10. Hirschowitz, A., Maggesi, M.: The algebraicity of the lambda-calculus. CoRR abs/0704.2900 (2007), http://arxiv.org/abs/0704.2900

11. Hirschowitz, A., Maggesi, M.: Modules over monads and initial semantics. Inf. Comput. 208(5), 545-564 (2010)

12. Hur, C.K.: Categorical equational systems: algebraic models and equational reasoning. Ph.D. thesis, University of Cambridge, UK (2010)

13. Hyland, J.M.E., Ong, C.H.: On full abstraction for PCF. Information and Computation 163, 285-408 (2000)

14. Plotkin, G.D.: LCF considered as a programming language. Theoretical Computer Science 5(3), 223-255 (1977) 